\title{
Biomarker heterogeneity between primary breast cancer and synchronous axillary lymph node metastases
}

\author{
XUN XI, XING-WEI HUANG, HUO-ZHONG YUAN, CHUN HE, JUN NI and FU-LAN YANG \\ Department of Thyroid and Breast Surgery, The People's Hospital of Ganzhou, \\ Ganzhou Affiliated Hospital of Nanchang University, Ganzhou, Jiangxi 341000, P.R. China
}

Received March 12, 2020; Accepted July 23, 2020

DOI: $10.3892 / \mathrm{ol} .2020 .12136$

\begin{abstract}
Whether the expression status of estrogen receptor (ER), progesterone receptor (PR), human epidermal growth factor receptor 2 (HER-2) receptor and $\mathrm{Ki}-67$ show concordance between the primary tumors and the synchronous axillary lymph node (ALN) metastases has been discussed in numerous studies. However, to date, the results of these studies remain controversial. Therefore, the present study aimed to investigate whether the expression of ER, PR, HER-2 and $\mathrm{Ki}-67$ was in concordance between the primary tumors and synchronous ALN metastases in patients with operable breast cancer (BC). A total of 60 tissue samples were collected from patients with primary operable BC diagnosed with primary tumors and synchronous ALN metastases. The expression levels of the four biomarkers, ER, PR, HER-2 and Ki-67, were assessed in primary lesions and synchronous ALN metastases samples using immunohistochemistry. The cut-off values were set to $10 \%$ for ER and PR, while the labeling index of $\mathrm{Ki}-67$ was set to $14 \%$. The immunostaining intensity of ER and PR was scored as negative (-), $1+, 2+$ and $3+$. The criteria for HER-2 testing in BC were implemented according to the American Society of Clinical Oncology (ASCO) and the College of American Pathologists (CAP) guidelines. The concordance rates for ER, PR and HER-2 were 96.7 (58/60), 96.7 (58/60) and 90\% (54/60), respectively. In addition, the kappa values of consistency in the primary lesions and the synchronous ALN metastases were 0.773 for ER, 0.654 for PR and 0.785 for HER-2. Furthermore, the P-values of ER, PR and $\mathrm{Ki}-67$ numerical variables between the two groups were $0.393,0.400$ and 0.331 , respectively, as demonstrated using a non-parametric Wilcoxon signed rank test. The findings of
\end{abstract}

Correspondence to: Professor Xing-Wei Huang, Department of Thyroid and Breast Surgery, The People's Hospital of Ganzhou, Ganzhou Affiliated Hospital of Nanchang University, 16 Meiguan Avenue, Zhanggong, Ganzhou, Jiangxi 341000, P.R. China E-mail: xixun2010@163.com

Key words: breast cancer, axillary lymph node, estrogen receptor, progesterone receptor, human epidermal growth factor receptor-2, Ki-67 the present study demonstrated a high degree of concordance between the expression of ER, PR, HER-2 and Ki-67 in the primary tumors and that in the synchronous ALN metastases, suggesting that the BC primary tumor biomarkers may be used for the prognosis of synchronous ALN metastases in patients with operable BC.

\section{Introduction}

Breast cancer (BC) is classified into five intrinsic subtypes according to gene expression profiling obtained by DNA microarrays $(1,2)$. Since the 2011 St Gallen symposium, a surrogate classification of intrinsic subtypes based on a four BC immunohistochemical (IHC) biomarker panel, namely estrogen receptor (ER), progesterone receptor (PR), human epidermal growth factor receptor 2 (HER-2) receptor and $\mathrm{Ki}-67$, has been in use (3). Therefore, BC has begun to enter the era of precision treatment. Molecular phenotypes may not only determine the $\mathrm{BC}$ treatment approach, but also predict the prognosis of patients; therefore, the accurate detection of these four molecular markers is necessary. It is widely accepted that the expression of the hormone receptors, ER and PR, determine the endocrine therapy approach in order to inhibit the recurrence and metastasis of BC. Additionally, both molecules exhibit a good prognostic index $(4,5)$. In clinical practice, HER-2-positive patients are prone to recurrence and metastasis; therefore, anti-HER-2 targeted therapy is commonly applied according to the National Comprehensive Cancer Network (NCCN) guidelines (6-8). Additionally, Ki-67 index exhibits a prognostic value in $\mathrm{BC}$. Therefore, increased $\mathrm{Ki}-67$ index value is associated with a poorer prognosis. Ki-67 protein is expressed in cell nuclei during cell cycle phases G1, S and M. In clinical practice, usually only the biomarkers for primary tumors are used to predict primary tumors accompanied by synchronous axillary metastases (9). However, whether the expression of theses biomarkers is in concordance between the $\mathrm{BC}$ primary tumor and synchronous ALN metastases remains controversial.

Therefore, the present prospective study aimed to investigate whether the expression of four BC biomarkers (ER, PR, HER-2 and Ki-67) in the primary tumors was in accordance with that in the synchronous ALN metastases, to provide a more accurate indicator for the clinical treatment and prognosis of patients with $\mathrm{BC}$. 


\section{Patients and methods}

Patients. A total of 60 female patients with BC, aged between 27 and 68 years old (median age, 45 years), admitted to the People's Hospital of Ganzhou (Ganzhou, China) between June 2017 and June 2018, were enrolled in the present study. The inclusion criteria were as follows: i) pathological diagnosis of invasive ductal carcinoma; ii) early $\mathrm{BC}$ with a single lesion in the primary tumor; and iii) with at least one positive axillary lymph node (ALN; $>2.0 \mathrm{~mm}$ ). The exclusion criteria for all study subjects were as follows: i) postoperative specimens from palliative surgery; ii) with neoadjuvant chemotherapy; iii) a primary tumor with multifocal/multicenter lesions; and iv) accompanied by distant metastases.

Immunohistochemistry (IHC). The expression profiles of ER, PR, HER-2 and Ki-67 in the primary tumors and synchronous ALN metastases were determined using immunohistochemistry (IHC). The tissues were fixed in $10 \%$ neutral buffered formalin at room temperature for 24-48 $\mathrm{h}$ and were embedded in paraffin. Tissue sections (4- $\mu \mathrm{m}$-thick) were deparaffinized in xylene at $37^{\circ} \mathrm{C}$ for $10 \mathrm{~min}$ and rehydrated in 100,96 and $70 \%$ ethanol. Endogenous peroxidase was blocked by incubation in $3 \% \mathrm{H}_{2} \mathrm{O}_{2}$ in methanol at $37^{\circ} \mathrm{C}$ for $10 \mathrm{~min}$, followed by rinsing in PBS. Sections were then subjected to antigen retrieval by immersion in citrate buffer $(\mathrm{pH} 6.0)$ preheated to $99^{\circ} \mathrm{C}$ for $40 \mathrm{~min}$. The sections were incubated with primary antibodies (all ready to use) against ER (clone SP1; cat. no. 790-4325; Ventana Medical Systems, Inc.),PR (clone 1E2; cat. no. 790-4296; Ventana Medical Systems, Inc.), HER-2 (C-erbB-2; clone 4B5; cat. no. 790-2991; Ventana Medical Systems, Inc.) and Ki-67 (cat. no. MAB-0672; Fuzhou Maixin Biotech Co., Ltd.). Blots were developed using the labeled streptavidin biotinylated antibody (ready to use; cat no. 760-500; Roche Diagnostics) at $37^{\circ} \mathrm{C}$ for $8 \mathrm{~min}$ using a Ventana Benchmark XT automated immunostaining system (Roche Diagnostics) according to the manufacturer's protocol. IHC results of ER (Fig. 1), PR (Fig. 2), HER-2 (Fig. 3) and Ki-67 (Fig. 4C) were visualized and imaged using the ZEISS Axio Lab A1 light microscope at a magnification of x200.

The cut-off level of ER and PR positively stained cell nuclei was set to $\geq 10 \%$. The expression intensity of ER and PR was scored on a scale of '-' to '+++', where '-', '+', '++' and ' +++ ' indicated $0,0-25,26-50$ and $>50 \%$ positive tumor cells, respectively. HER-2 IHC staining was scored as $0,1+$, $2+$ or $3+$. HER-2 scores of 0 and $1+$ were considered negative, while a score of $3+$ was considered positive. When a HER-2 score of 2+ was obtained, the results were reassessed using fluorescence in situ hybridization (FISH). FISH (FP-001; HER-2/CEP17 dual-color probe mixture; Wuhan Healthcare Biotechnology Co., Ltd.) assay was performed according to the American Society of Clinical Oncology (ASCO) and the College of American Pathologists (CAP) guidelines (10). FISH analysis of HER-2 (Fig. 4A and B) was visualized and imaged using the LEICA DM2500 fluorescence microscope at a magnification of $x 600$. The Ki-67 index values are expressed as the percentage of tumor cells with positively stained nuclei and the cut-off point was set to $14 \%$ (11).

Statistical analyses. The consistency of the expression ER, PR and HER-2 biomarkers between the primary tumors and ALN
Table I. Breast cancer subtypes in primary tumors and paired ALN metastases.

\begin{tabular}{lcc}
\hline HER-2 status & $\begin{array}{c}\text { Primary } \\
\text { tumor }\end{array}$ & $\begin{array}{c}\text { ALN } \\
\text { metastases }\end{array}$ \\
\hline HER-2-positive status (n=22) & & \\
ER+/PR+ & 14 & 14 \\
ER+/PR- & 3 & 1 \\
ER-/PR- & 5 & 7 \\
ER-/PR+ & 0 & 0 \\
HER-2-negative status (n=38) & & \\
ER+/PR+ & 25 & 23 \\
ER+/PR- & 1 & 3 \\
ER-/PR- & 12 & 12 \\
ER-/PR+ & 0 & 0 \\
\hline
\end{tabular}

ER, estrogen receptor; PR, progesterone receptor; HER-2, human epidermal growth factor receptor 2; ALN, axillary lymph node.

metastases was analyzed using $\chi^{2}$ test. The concordance of ER, PR $(-,+,++,+++)$ and HER-2 (negative, positive) expression intensity between the primary tumors and ALN metastases was analyzed using Kappa test. When the values for ER, PR and Ki-67 expression in the two groups were not normally distributed, a non-parametric Wilcoxon signed rank test was performed. $\mathrm{P}<0.05$ was considered to indicate a statistically significant difference. All statistical analyses were performed using the SPSS 20.0 software (IBM Corp.).

\section{Results}

Concordance/discordance in the expression of ER. The different BC subtypes in the primary tumors and ALN metastases are presented in Table I. The positive expression rate of ER in the primary tumors and ALN metastases in Table II was $71.7(43 / 60)$ and $68.3 \%$ (41/60), respectively. In addition, the negative ER expression rate was $28.3 \%$ (17/60) in the primary tumors and $31.7 \%$ (19/60) in ALN metastases. The P-value obtained when comparing ER expression between primary tumors and ALN metastases was not significant $(\mathrm{P}=0.393$; Table III). The kappa value obtained when comparing ER expression intensity in primary tumors with that in ALN metastases was 0.773 , thereby indicating a high degree of consistency in ER expression (Table IV). Finally, as shown in Table V, the concordance and discordance rates for ER were $96.7(58 / 60)$ and $3.33 \%(2 / 60)$, respectively. In the two discordance cases, both samples were positive for ER in the primary tumors and negative in the ALN metastases.

Concordance/discordance in the expression of $P R$. The expression of PR was determined in the primary tumors and ALN metastases using IHC. The PR-positive and -negative rates in primary tumors were $65(39 / 60)$ and $35 \%(21 / 60)$, respectively. Consistent with that in the primary tumors, the PR-positive rate in ALN metastases was 61.7\% (37/60) and the negative rate was $38.3 \%$ (23/60); (Table II). Furthermore, the P-value 
Table II. Expression status of biomarkers in the primary tumors and ALN metastases.

\begin{tabular}{lll}
\hline Biomarker & Primary tumor & ALN metastases \\
\hline ER & & \\
Positive rate $(\%)$ & $43 / 60(71.7)$ & $41 / 60(68.3)$ \\
Negative rate $(\%)$ & $17 / 60(28.3)$ & $19 / 60(31.7)$ \\
PR & & \\
Positive rate (\%) & $39 / 60(65)$ & $37 / 60(61.7)$ \\
Negative rate $(\%)$ & $21 / 60(35)$ & $23 / 60(38.3)$ \\
HER-2 & & \\
Positive rate $(\%)$ & $22 / 60(36.7)$ & $22 / 60(36.7)$ \\
Negative rate $(\%)$ & $38 / 60(63.3)$ & $38 / 60(63.3)$ \\
\hline
\end{tabular}

The consistency of biomarker expression between the primary tumors and the paired ALN was analyzed by $\chi^{2}$ test. ER, estrogen receptor; PR, progesterone receptor; HER-2, human epidermal growth factor receptor 2; ALN, axillary lymph node.

Table III. ER, PR and Ki-67 numerical expression variables.

\begin{tabular}{lccrc}
\hline Marker & Primary tumor & ALN metastases & Z & P-value \\
\hline ER & $70.00(0.00-90.00)$ & $62.50(0.00-90.00)$ & -0.854 & 0.393 \\
PR & $20.00(0.00-50.00)$ & $6.50(0.00-50.00)$ & -0.842 & 0.400 \\
Ki-67 & $30.00(0.00-40.00)$ & $30.00(15.25-40.00)$ & -0.973 & 0.331 \\
\hline
\end{tabular}

P-values were obtained by non-parametric Wilcoxon signed rank test. ER, estrogen receptor; PR, progesterone receptor; ALN, axillary lymph node.

Table IV. Expression intensity and consistency of ER and PR in primary tumors and ALN metastases $(n=60)$.

\begin{tabular}{lrrrrr}
\hline & \multicolumn{5}{c}{ ALN metastases } \\
\cline { 2 - 5 } Primary & - & + & ++ & +++ & Kappa \\
\cline { 2 - 5 } & & & & & 0.773 \\
ER & 17 & 0 & 0 & 0 & \\
- & 1 & 7 & 3 & 0 & \\
+ & 1 & 2 & 8 & 2 & \\
++ & 0 & 0 & 1 & 18 & \\
+++ & & & & & 0.654 \\
PR & 1 & 10 & 2 & 0 & \\
- & 1 & 8 & 9 & 1 & \\
+ & 0 & 1 & 1 & 5 & \\
++ & & & & & 0.785 \\
+++ & 35 & 3 & & & \\
HER-2 & 3 & 19 & & & \\
- & & 0 & 0 & 0 & \\
+ & & & & & \\
\hline
\end{tabular}

ER, estrogen receptor; PR, progesterone receptor; HER-2, human epidermal growth factor receptor 2; ALN, axillary lymph node; HER-2 +/-, HER-2 positive/negative expression.

obtained when comparing PR expression between primary tumors and ALN metastases did not indicate a statistically
Table V. Changes in the expression of the biomarkers between primary BC tumors and ALN metastases.

\begin{tabular}{lllll}
\hline & \multicolumn{2}{c}{ Primary BC } & & \multicolumn{2}{c}{ ALN metastases } \\
\cline { 2 - 2 } \cline { 5 - 5 } Biomarker & Discordance rate $(\%)$ & & $(+) \rightarrow(-)$ & $(-) \rightarrow(+)$ \\
\hline ER & $2 / 60(3.33)$ & & 2 & 0 \\
PR & $2 / 60(3.33)$ & & 2 & 0 \\
HER-2 & $6 / 60(10)$ & 3 & 3 \\
\hline
\end{tabular}

ER, estrogen receptor; PR, progesterone receptor; HER-2, human epidermal growth factor receptor 2; ALN, axillary lymph node; BC, breast cancer; +/-, positive/negative ER, PR and HER-2 expression.

significant difference $(\mathrm{P}=0.400$; Table III). As shown in Table IV, the kappa value of consistency was 0.654 for PR expression in primary lesions and ALN metastases, suggesting a high degree of consistency in its expression. Consistent with the ER IHC results, the concordance and discordance rates for PR expression in Table V were 96.7 (58/60) and 3.33\% (2/60), respectively. In the two discordance cases, all samples were positive for PR expression in the primary tumor but negative in the ALN metastases.

Concordance/discordance in the expression of HER-2. The expression of HER-2 was investigated. The results revealed 

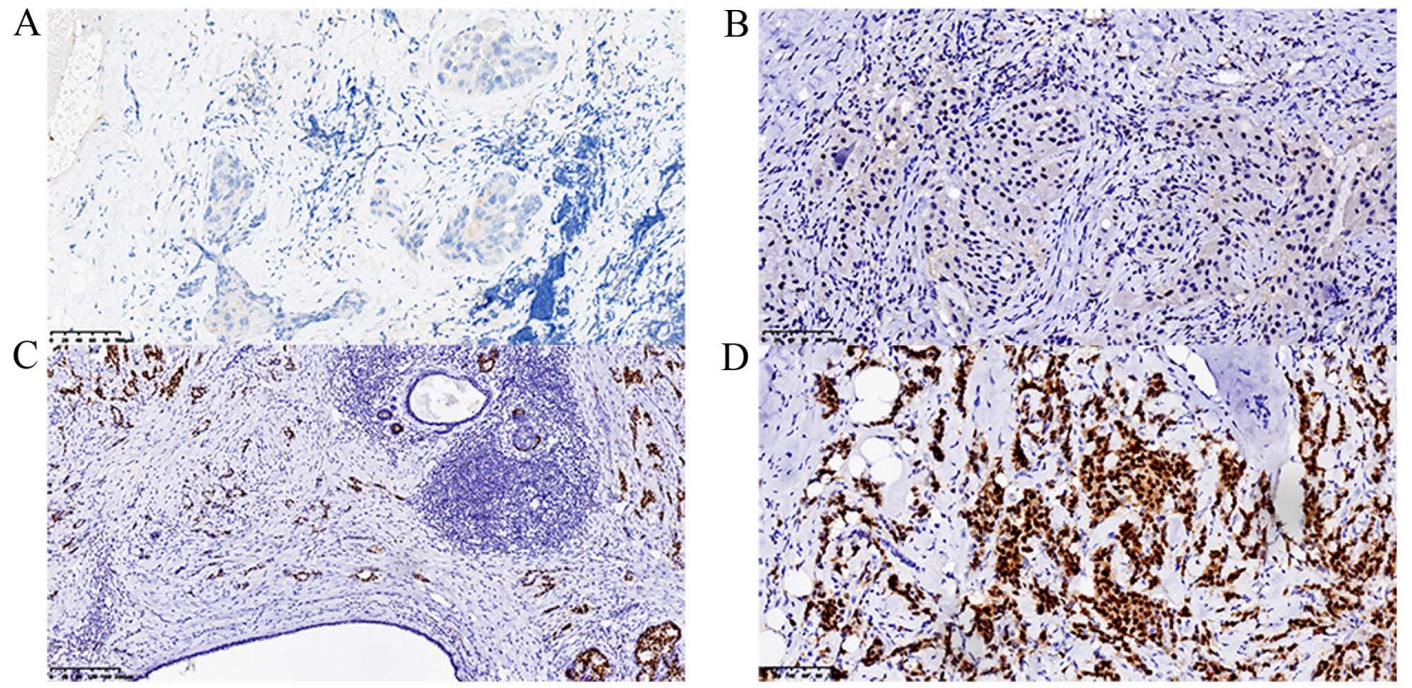

Figure 1. Immunohistochemistry images of estrogen receptor staining. Images represent the tumor staining scores (A) '-', (B) '+', (C) '++' and (D) '+++' (magnification, $\mathrm{x} 200$ ).
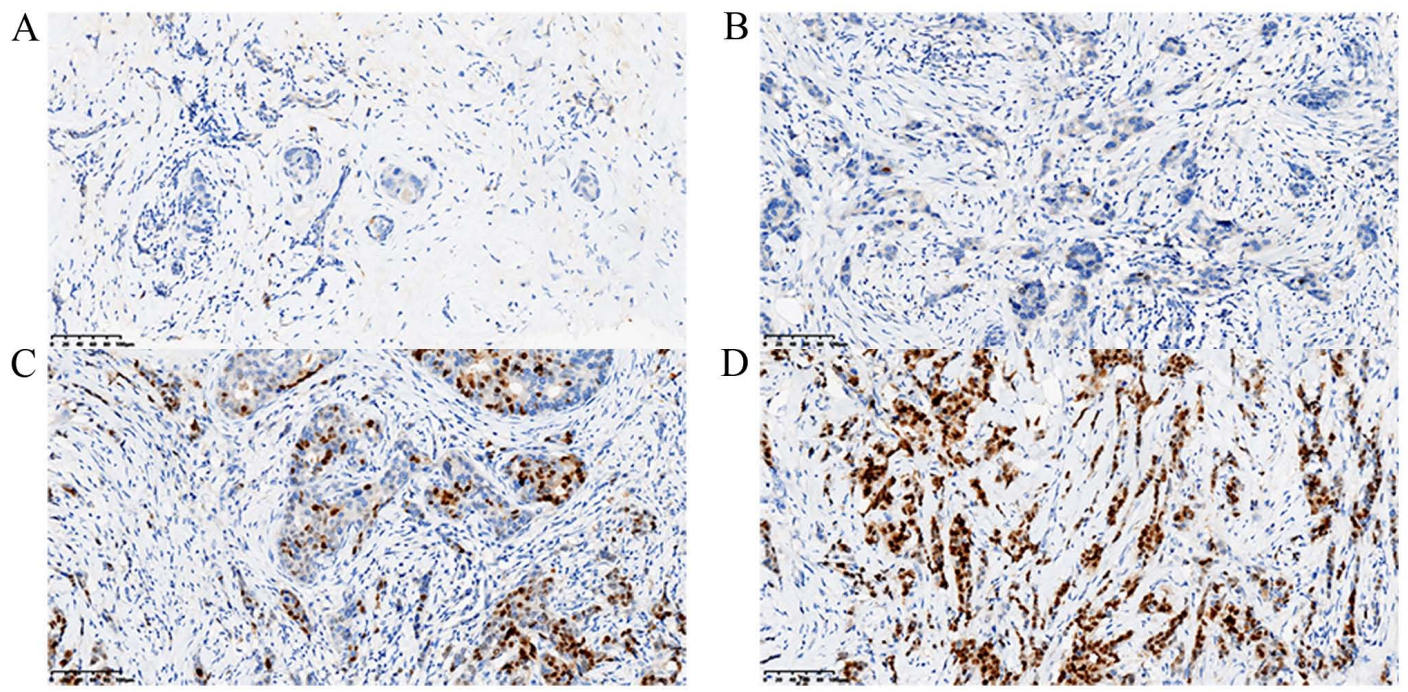

Figure 2. Immunohistochemistry images of progesterone receptor staining. Images represent the tumor staining scores (A) ' - ', (B) ' + ', (C) '++' and (D) '+++' (magnification, x200).

that the HER-2-negative rate in the primary tumors and ALN metastases was $63.3 \%(38 / 60)$ in the two groups (Table II). The kappa value of consistency for HER-2 was 0.785 , indicating a high degree of consistency for HER-2 expression between the primary tumors and ALN metastases (Table IV). Finally, the concordance rate for HER-2 was $90 \%(54 / 60)$ and the discordance rate was $10 \%$ (6/60; Table V). Among the six discordance cases, three were positive for HER-2 in the primary tumor and negative in the ALN metastases, while three were negative in the primary tumor and positive in ALN metastases.

Concordance/discordance in the expression of $\mathrm{Ki}$-67. Analysis of the P-values comparing Ki-67 expression between primary tumors and ALN metastases $(\mathrm{P}=0.331)$, using a non-parametric Wilcoxon signed rank test, suggested that the Ki-67 expression in the primary tumors was consistent with that in the ALN metastases (Table III).

\section{Discussion}

ALN metastasis is very common in BC. Whether the expression of the biomarkers in the primary tumors is in concordance with that in the synchronous ALN metastases remains controversial $(12,13)$. It has been reported that the discordance rates in the primary tumors and recurrent/metastatic lesions were 10-30\% for ER, 25-55\% for PR and 10-15\% for HER-2 (14-18). Other studies $(19,20)$ have investigated the discordance in biomarker expression between the circulating tumor cells and the primary tumors. Furthermore, several retrospective studies have revealed discrepancies in ER, PR, Ki-67 and HER-2 expression between primary breast tumors and synchronous ALN metastases (21-24). Nedergaard et al (21) investigated the ER expression status in 101 primary BC tumors and the paired ALN metastases. The discordant rate for ER was $21 \%$ between the primary tumors and the corresponding metastatic lesions (21). Accordingly, Aitken et al (22) identified 

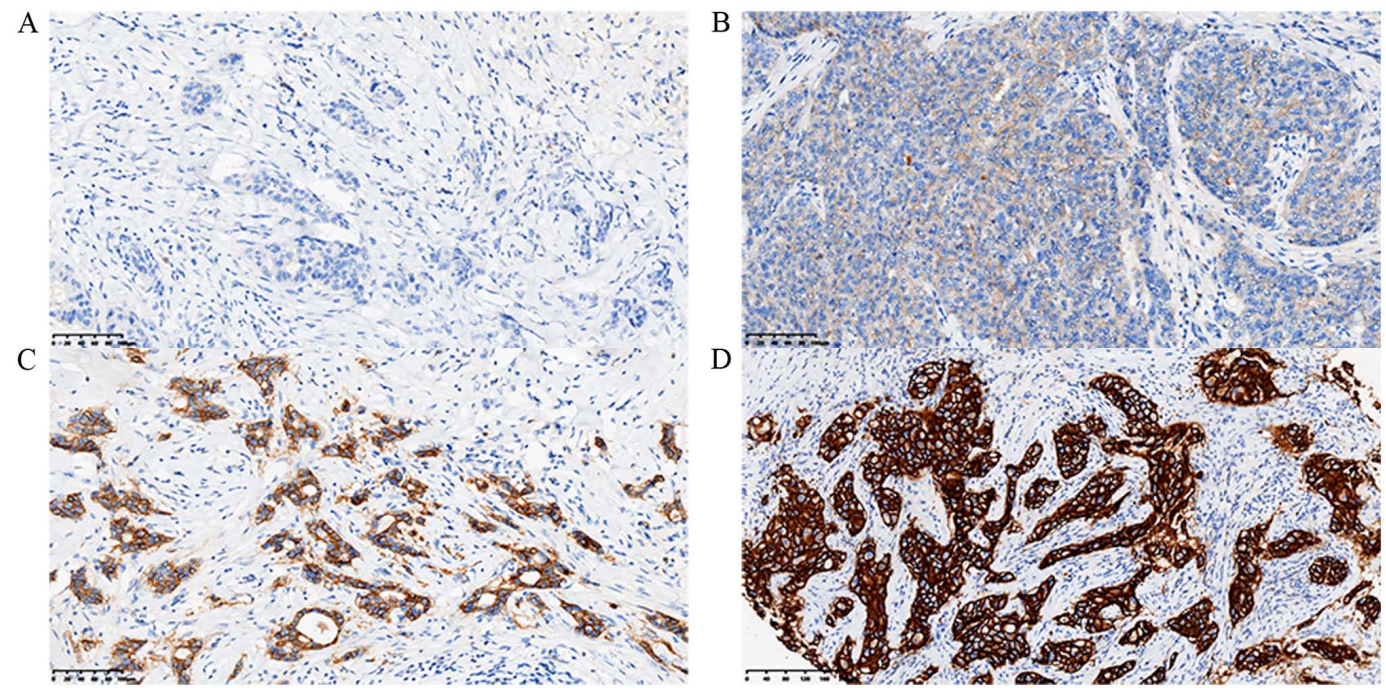

Figure 3. Immunohistochemistry images of human epidermal growth factor receptor 2 staining. Images represent the tumor staining scores (A) 0 , (B) $1+$, (C) 2+ and (D) 3+ (magnification, x200).
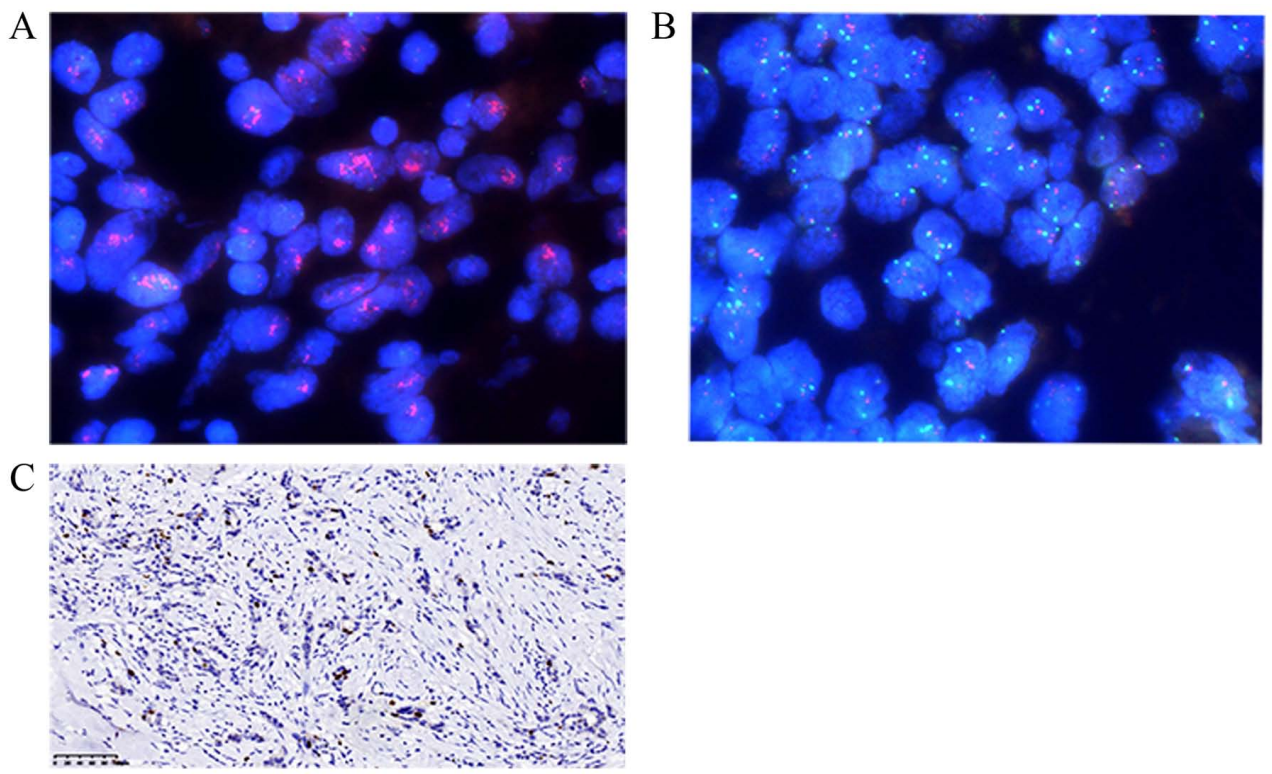

Figure 4. FISH of HER-2 and immunohistochemistry image of Ki-67 staining. (A) Positive and (B) negative FISH of HER-2 (magnification, x600). (C) Immunohistochemistry image of Ki-67 staining (magnification, x200). FISH, fluorescence in situ hybridization; HER-2, human epidermal growth factor receptor 2 .

the changes in ER, PR and HER-2 expression between the primary tumors and synchronous ALN metastases. The results revealed that the discrepancy rates for ER, PR and HER-2 were 28.9 (55/190), 23.7 (45/190) and 8.9\% (17/190), respectively (22). Additionally, Feng et al (23) investigated the expression of ER and HER-2 in primary tumors and ALN metastases in 80 patients with invasive ductal BC. The discordance rates in the present study were $8.8 \%$ for ER and $11.3 \%$ for HER-2 (23). Kinoe et al (24) reported that the discordance rates between the primary tumors and the lymph node metastases were $28.8 \%$ for ER (positive in primary tumors and negative in lymph nodes or negative in primary tumors and positive in lymph nodes, $22.1 / 6.7 \%), 31.7 \%$ for PR (26.9/4.8\%), 13.5\% for HER-2 (12.5/1.0\%) and $43.3 \%$ for Ki-67 (high in primary tumors and low in lymph nodes or low in primary tumors and high in lymph nodes, $12.5 / 30.8 \%$ ). Overall, the aforementioned studies revealed increased discordance rates in ER and PR expression and decreased discordance in HER-2 expression. Furthermore, the results of these studies suggested that the most common changes in patients with breast cancer were hormone receptor and HER-2 positive in primary tumors, but negative in synchronous ALN metastases. Due to these discrepancies, researchers have suggested that the determination of biomarker expression should be performed simultaneously in the synchronous ALN metastases and their corresponding primary tumors (25-27).

However, other studies have demonstrated that the expression profile of the molecular markers was consistent between the primary and metastatic lesions $(9,19,28)$. Consistent with the results of the present study, Zhao et al (9) identified 
that the concordance rates between the $\mathrm{BC}$ primary tumors and ALN metastases were 72.2\% (39/54) for ER, 88.9\% (48/54) for PR and 90.7\% (49/54) for HER-2. Therefore, no statistically significant differences were observed (9). However, in contrast to this previous study, patients that had undergone neoadjuvant therapy were not included in the present study.

Aktas et al (19) demonstrated that the primary tumors and circulating tumor cells displayed a concordant HER-2, ER and $P R$ expression status in $59(\mathrm{P}=0.262), 39(\mathrm{P}=0.51)$ and $44 \%(\mathrm{P}=0.62)$ of cases, respectively (19). Furthermore, D'Andrea et al (28) studied the expression of 10 biomarkers in 90 pure invasive ductal carcinomas with 10 or more ALNs involved and without evidence of distant metastasis. This study revealed a close association between the primary tumors and the corresponding metastatic nodes in terms of the expression of all 10 biomarkers investigated (28). However, in the aforementioned study, the authors did not investigate the expression of ER, PR and HER-2 in order to compare their expression values between the primary tumors and the paired metastatic nodes.

In the present study, the consistency rate of ER and PR expression was $96.7 \%$ (58/60). Only two cases with primary tumors positive for ER and PR exhibited negative expression in the ALN metastases, while only one case with primary tumors negative for ER and PR expression exhibited positive expression in the ALN metastases. In addition, the concordance rate for HER-2 was $90 \%(54 / 60)$ and only six cases (10\%) exhibited discordance. Among the six cases, three were HER-2-negative in the primary tumors and positive in the ALN metastases, while three were HER-2-positive in the primary tumors but negative in the ALN metastases. Furthermore, the present study compared the expression intensity of ER, PR and HER-2, as well as the numeric expression variables of ER, PR and Ki-67, between the primary tumors and the corresponding synchronous ALN metastases. Therefore, the kappa values of consistency for ER, PR and HER-2 expression intensity between the primary lesions and the synchronous ALN metastases were $0.773,0.654$ and 0.785 , respectively, thereby demonstrating a high degree of consistency. In addition, the P-values comparing ER, PR and Ki-67 expression variables between the two groups were $0.393,0.400$ and 0.331 , respectively, as verified using a non-parametric Wilcoxon signed rank test. Therefore, no statistically significant differences were observed.

However, some studies have suggested that the concordance between primary lesions and metastases is associated with a poorer prognosis (16). Jung et al (29) demonstrated that the overall survival time was longer in patients with $\mathrm{BC}$ with concordant hormone receptor expression between the primary breast tumors and brain lesions, compared with patients exhibiting discordant expression patterns. However, the difference was not statistically significant (discordant vs. concordant median survival time, 19.2 vs. 31.1 months; $\mathrm{P}=0.181$ ) (29). Furthermore, other previous studies reported that neoadjuvant/adjuvant chemotherapy, and endocrine and targeted therapy may affect the expression of the biomarkers in the primary and metastatic lesions $(16,18)$. In the present study, patients undergoing chemotherapy, endocrine therapy or targeted therapy were excluded, and only early operable patients were enrolled, which may explain the high consistency of our findings. Sighoko et al (30) applied a Bayesian misclassification correction method on data on hormone receptor expression status from two primary BCs from the Surveillance, Epidemiology and End Results (SEER) database between 1990 and 2010 and on data on primary and paired recurrent or metastatic disease assembled from a meta-analysis of 36 studies. The authors suggested a considerable proportion of discordance in hormone receptor expression status that should be attributed to misclassification in receptor assessment (30).

In conclusion, the findings of the present study indicated that the ER, PR, HER-2 and Ki 67 expression status was in concordance between the primary breast tumors and synchronous ALN metastases. Therefore, molecular biomarkers of the primary tumors could be also used as biomarkers for synchronous axillary metastases in some patients. However, the application of these biomarkers in the lymph nodes should be further evaluated due to the lack of high-quality, large-scale prospective studies or high-quality meta-analysis.

\section{Acknowledgements}

Not applicable.

\section{Funding}

The present study was supported by the Guiding Science and Technology Program Project in Ganzhou City, Jiangxi, China (grant no. GZ2017ZSF199).

\section{Availability of data and materials}

All data generated and/or analyzed during this study are included in this article.

\section{Authors' contributions}

XX and XWH conceived and designed the study. FLY was responsible for the acquisition of data. JN performed the statistical analysis. HZY and $\mathrm{CH}$ analysed and interpreted the data, drafted the manuscript and revised it critically for important intellectual content. All authors read and approved the final manuscript.

\section{Ethics approval and consent to participate}

Written informed consent was obtained from all patients for the use of their tissues for research purposes, and the study protocol was approved by Ganzhou People's Hospital Ethics Committee (Ganzhou, China).

\section{Patient consent for publication}

Not applicable.

\section{Competing interests}

The authors declare that they have no competing interests.

\section{References}

1. Prat A and Perou CM: Deconstructing the molecular portraits of breast cancer. Mol Oncol 5: 5-23, 2011. 
2. Cancer Genome Atlas Network: Comprehensive molecular portraits of human breast tumours. Nature 490: 61-70, 2012.

3. Gándara-Cortes M,Vázquez-Boquete Á,Fernández-Rodríguez B, Viaño P, Ínsua D, Seoane-Seoane A, Gude F, Gallego R, Fraga M, Antúnez JR, et al: Breast cancer subtype discrimination using standardized 4-IHC and digital image analysis. Virchows Arch 472: 195-203, 2018.

4. Subik K, Lee JF, Baxter L, Strzepek T, Costello D, Crowley P, Xing L, Hung MC, Bonfiglio T, Hicks DG and Tang P: The expression patterns of ER, PR, HER2, CK5/6, EGFR, Ki-67 and AR by immunohistochemical analysis in breast cancer cell lines. Breast Cancer (Auckl) 4: 35-41, 2010.

5. Voduc KD, Cheang MC, Tyldesley S, Gelmon K, Nielsen TO and Kennecke H: Breast cancer subtypes and the risk of local and regional relapse. J Clin Oncol 28: 1684-1691, 2010.

6. Gradishar WJ, Anderson BO, Balassanian R, Blair SL, Burstein HJ, Cyr A, Elias AD, Farrar WB, Forero A Giordano SH, et al: Invasive breast cancer version 1.2016, NCCN clinical practice guidelines in oncology. J Natl Compr Canc Netw 14: 324-354, 2016.

7. Mohsenifar J, Almassi-Aghdam M,Mohammad-Taheri Z,Zare K, Jafari B, Atri M, Mortazavi SH, Azadeh P, Bagherzadeh M, Azargashb E and Rahimi F: Prognostic values of proliferative markers ki-67 and repp86 in breast cancer. Arch Iran Med 10 27-31, 2007.

8. Kontzoglou K, Palla V, Karaolanis G, Karaiskos I, Alexiou I, Pateras I, Konstantoudakis K and Stamatakos M: Correlation between Ki67 and breast cancer prognosis. Oncology 84 219-225, 2013

9. Zhao S, Xu L, Liu W, Lv C, Zhang K, Gao H, Wang J and Ma R: Comparison of the expression of prognostic biomarkers between primary tumor and axillary lymph node metastases in breast cancer. Int J Clin Exp Pathol 8: 5744-5748, 2015.

10. Wolff AC, Hammond ME, Hicks DG, Dowsett M, McShane LM, Allison KH, Allred DC, Bartlett JM, Bilous M, Fitzgibbons $\mathrm{P}$, et al: Recommendations for human epidermal growth factor receptor 2 testing in breast cancer: American society of clinical oncology/college of American pathologists clinical practice guideline update. J Clin Oncol 31: 3997-4013, 2013.

11. Goldhirsch A, Winer EP, Coates AS, Gelber RD, Piccart-Gebhart M, Thürlimann B and Senn HJ; Panel members: Personalizing the treatment of women with early breast cancer: Highlights of the St Gallen international expert consensus on the primary therapy of early breast cancer 2013. Ann Oncol 24: 2206-2223, 2013.

12. Yao ZX, Lu LJ, Wang RJ, Jin LB, Liu SC, Li HY, Ren GS, Wu KN, Wang DL and Kong LQ: Discordance and clinical significance of ER, PR, and HER2 status between primary breast cancer and synchronous axillary lymph node metastasis. Med Oncol 31: 798, 2014

13. Song JL, Chen C, Yuan JP and Sun SR: Progress in the clinical detection of heterogeneity in breast cancer. Cancer Med 5: 3475-3488, 2016.

14. Liedtke C, Broglio K, Moulder S, Hsu L, Kau SW, Symmans WF, Albarracin C, Meric-Bernstam F, Woodward W, Theriault RL, et al: Prognostic impact of discordance between triple-receptor measurements in primary and recurrent breast cancer. Ann Oncol 20: 1953-1958, 2009.

15. Sari E, Guler G, Hayran M, Altundag K, Gullu I and Ozisik Y: Comparison of ER, PR, HER2 in primary and paired relapsed/metastatic lesions of metastatic breast cancer patients. J Clin Oncol 27: 1063, 2009.

16. Niikura N, Liu J, Hayashi N, Mittendorf EA, Gong Y, Palla SL, Tokuda Y, Gonzalez-Angulo AM, Hortobagyi GN and Ueno NT: Loss of human epidermal growth factor receptor 2 (HER2) expression in metastatic sites of HER2-overexpressing primary breast tumors. J Clin Oncol 30: 593-599, 2012.
17. Dieci MV, Barbieri E, Piacentini F, Ficarra G, Bettelli S, Dominici M, Conte PF and Guarneri V: Discordance in receptor status between primary and recurrent breast cancer has a prognostic impact: A single-institution analysis. Ann Oncol 24: 101-108, 2013.

18. Curtit E, Nerich V, Mansi L, Chaigneau L, Cals L, Villanueva C, Bazan F, Montcuquet P, Meneveau N, Perrin S, et al: Discordances in estrogen receptor status, progesterone receptor status, and HER 2 status between primary breast cancer and metastasis. Oncologist 18: 667-674,2013

19. Aktas B, Kasimir-Bauer S, Müller V, Janni W, Fehm T, Wallwiener D, Pantel K and Tewes M; DETECT Study Group: Comparison of the HER2, estrogen and progesterone receptor expression profile of primary tumor, metastases and circulating tumor cells in metastatic breast cancer patients. BMC Cancer 16: 522, 2016.

20. Turner N, Pestrin M, Galardi F, De Luca F, Malorni L and Di Leo A: Can biomarker assessment on circulating tumor cells help direct therapy in metastatic breast cancer? Cancers (Basel) 6: 684-707, 2014.

21. Nedergaard L, Haerslev $T$ and Jacobsen GK: Immunohistochemical study of estrogen receptors in primary breast carcinomas and their lymph node metastases including comparison of two monoclonal antibodies. APMIS 103: 20-24, 1995.

22. Aitken SJ, Thomas JS, Langdon SP, Harrison DJ and Faratian D: Quantitative analysis of changes in ER, PR and HER2 expression in primary breast cancer and paired nodal metastases. Ann Oncol 21: 1254-1261, 2010

23. Feng T, Zhang J and Zhong L: Relationship of ER and HER-2 expression in primary tumor and axillary lymph node metastases of invasive ductal breast cancer. Chin J Gen Surg, 2012.

24. Kinoe H, Yamanouchi K, Kuba S, Morita M, Sakimura C, Kanetaka K, Takatsuki M, Abe K, Yano H, Matsumoto M, et al: Discordance of hormone receptor, human epidermal growth factor receptor-2, and $\mathrm{Ki}-67$ between primary breast cancer and synchronous axillary lymph node metastasis. J BUON 23: 60-66, 2018.

25. Santinelli A, Pisa E, Stramazzotti D and Fabris G: HER-2 status discrepancy between primary breast cancer and metastatic sites. Impact on target therapy. Int J Cancer 122: 999-1004, 2008.

26. Ataseven B, Gologan D, Gunesch A, Kehl V, Hoegel B, Beer M and Eiermann W: HER2/neu, topoisomerase 2a, estrogen and progesterone receptors: Discordance between primary breast cancer and metastatic axillary lymph node in expression and amplification characteristics. Breast Care (Basel) 7: 465-470, 2012.

27. Jensen JD, Knoop A, Ewertz M and Laenkholm AV: ER, HER2, and TOP2A expression in primary tumor, synchronous axillary nodes, and asynchronous metastases in breast cancer. Breast Cancer Res Treat 132: 511-521, 2012.

28. D'Andrea MR,Limiti MR,Bari M,Zambenedetti P, Montagutti A, Ricci F, Pappagallo GL, Sartori D, Vinante O and Mingazzini PL: Correlation between genetic and biological aspects in primary non-metastatic breast cancers and corresponding synchronous axillary lymph node metastasis. Breast Cancer Res Treat 101: 279-284, 2007

29. Jung J, Lee SH, Park M, Youn JH, Shin SH, Gwak HS and Yoo H Discordances in ER, PR, and HER 2 between primary breast cancer and brain metastasis. J Neurooncol 137: 295-302, 2018.

30. Sighoko D, Liu J, Hou N, Gustafson P and Huo D: Discordance in hormone receptor status among primary, metastatic, and second primary breast cancers: Biological difference or misclassification? Oncologist 19: 592-601, 2014.

This work is licensed under a Creative Commons Attribution-NonCommercial-NoDerivatives 4.0 International (CC BY-NC-ND 4.0) License. 